\title{
A HIGH-MASS, EIGHT-FOLD SYMMETRIC SILICON CARBIDE MEMS GYROSCOPE
}

Eugene Cook ${ }^{1 *}$, Michael Tomaino-Iannucci ${ }^{l}$, Jonathan Bernstein ${ }^{l}$, Marc Weinberg ${ }^{l}$, Jennifer Choy ${ }^{l}$, Karl Hobart ${ }^{2}$, Lunet Luna ${ }^{2}$, Marko Tadjer ${ }^{2}$, Rachael Myers-Ward ${ }^{2}$, Fritz Kub ${ }^{2}$, Yushi Yang ${ }^{3}$, Eldwin $_{\mathrm{Ng}^{3}}$, Ian Flader ${ }^{3}$, Yunhan Chen ${ }^{3}$, and Thomas Kenny ${ }^{3}$

${ }^{1}$ Draper, Cambridge, Massachusetts, USA

${ }^{2}$ U.S. Naval Research Laboratory, Washington DC, USA

${ }^{3}$ Stanford University, Stanford, California, USA

\section{ABSTRACT}

A novel MEMS gyroscope is presented, which blends the best aspects of traditional tuning fork gyroscopes with modern wholeangle capable designs such as rings and disks. The gyroscopes were fabricated in both silicon and silicon carbide. The resonators were measured to have quality factors of $120,000(\mathrm{SiC})$ and 260,000 (Si), and frequency splits of $0.08 \%(\mathrm{SiC})$ and $0.24 \%(\mathrm{Si})$. Open loop rate mode gyroscope operation was demonstrated on both gyroscopes.

\section{GYRO DESIGN}

The gyro incorporates large masses and displacements for high sensitivity and reduced Brownian motion (like a traditional tuningfork gyro), while also exhibiting a symmetric in-plane modal pattern commonly called " $n=2$ " and illustrated in Figure 4. Eight wedges are arranged on flexures and coupled together. The symmetric, Coriolis-coupled modes enable mode-reversal and parameteridentification-based in-situ calibration techniques, as well as wholeangle mode operation [1]. Electrostatic drive, sense, and tuning electrodes are incorporated for matched frequencies.

\section{FABRICATION}

Gyroscopes were built in silicon (Figure 3), using the epi-seal process [2]. Additionally, a new Silicon Carbide-based process (Figure 1) was developed to explore the performance benefits available from this novel material [3]. Silicon carbide offers superior material properties (Table 1) and superior material isoelasticity [4], leading to reduced material-driven asymmetry, important for whole-angle gyro operation. The process begins with wafer bonding and thinning to form a $40-\mu \mathrm{m}$ thick single crystal $4 \mathrm{H}$ Silicon Carbide-on-insulator (SiCOI) wafer. A nickel mask is used to deep reactive ion etch features with aspect ratio up to 10:1 into the device layer. Metal contact pads are formed and the devices are released in HF vapor. The finished devices (Figure 2) are the first bulk $\mathrm{SiC}$ resonators with traditional MEMS flexures and masses. (a)

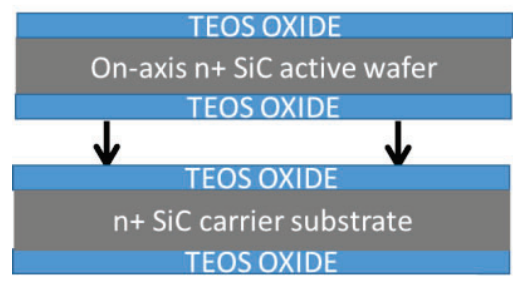

(b)

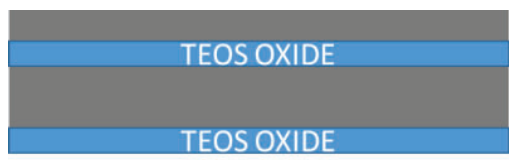

(c)

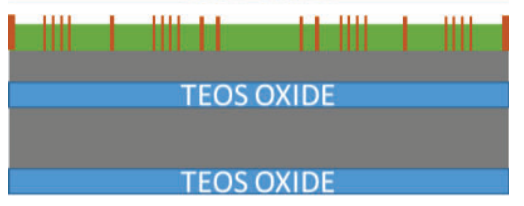

(d)

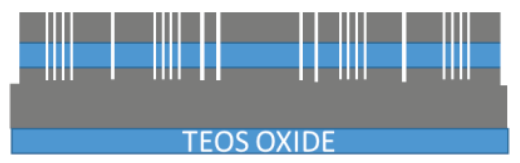

(e)

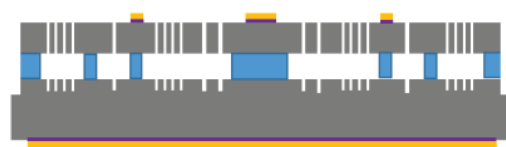

Figure 1: The silicon carbide on insulator (SiCOI) fabrication process for the gyro is illustrated, including wafer bond (a), thinning of the top layer (b), electroplated nickel mask (c), deep reactive ion etch (d), and vapor $H F$ release and metallization (e).

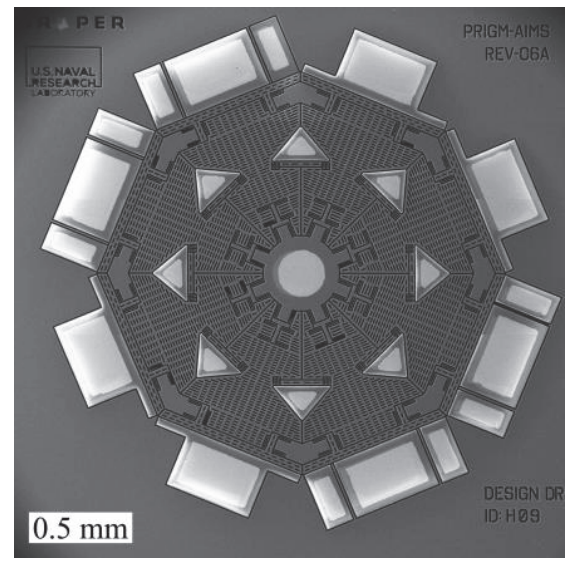

Figure 2: SEM showing a complete silicon carbide gyro with metal wirebond pads.

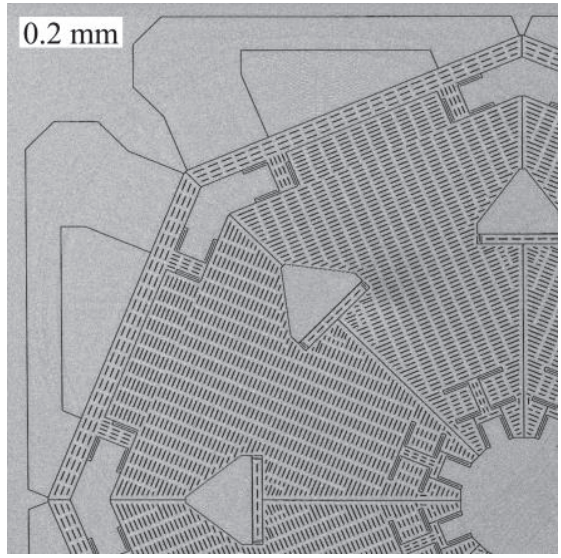

Figure 3: SEM showing details of a silicon gyro, including flexures and release slots.

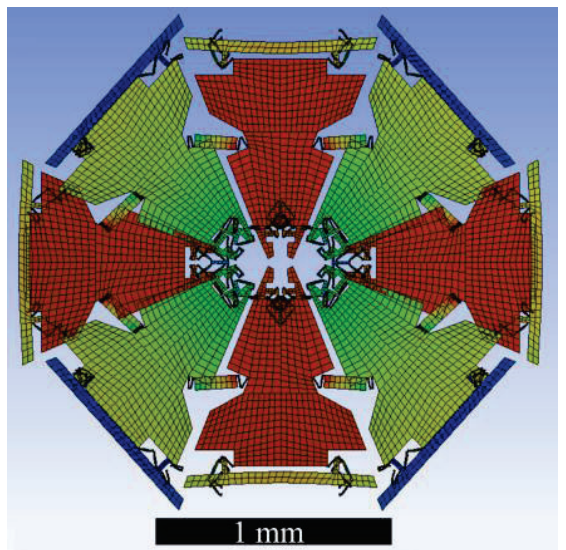

Figure 4: The gyro $n=2$ mode shape is simulated by the finite element method. 


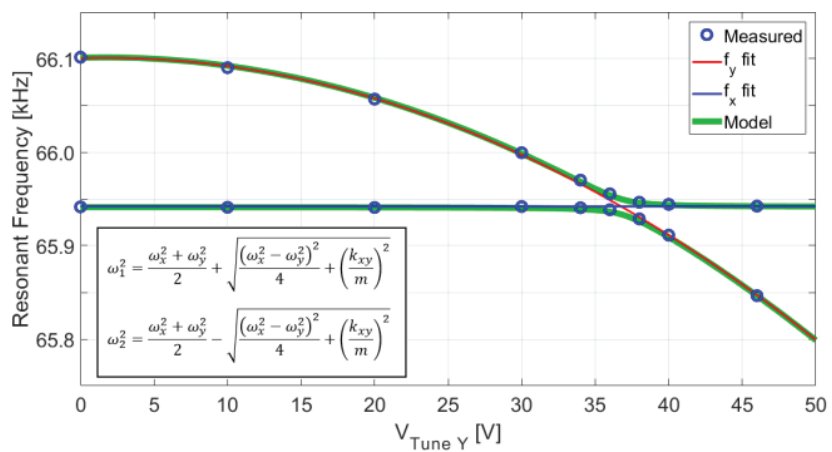

Figure 5: Electrostatic tuning of the silicon device to achieve matched vibratory modes is measured and modeled. The two modes are brought together with one of the on-axis tuning electrodes. Blue circles represent measured resonant frequencies at different voltages applied to one of the tuning electrodes. Green curves are the modeled results according to the formulae. Between 30 and 40 volts, the nonzero cross-axis term $\left(k_{x y} / \mathrm{m}\right.$, estimated at $\left.4.2 \times 10^{7} \mathrm{~s}^{-2}\right)$ prevents perfect tuning. The cross-axis tuning electrode would be used to tune out $k_{x y}$ and remove the residual split.

\section{RESULTS}

Resonators with $\mathrm{n}=2$ frequencies from 20 to $150 \mathrm{kHz}$ were built. Quality factors (Q) of 120,000 and 260,000 were measured in vacuum for the $88 \mathrm{kHz} \mathrm{SiC}$ and $65.9 \mathrm{kHz}$ Si resonators, respectively. $\mathrm{Q}$ is limited by thermoelastic damping (TED):

where thermoelastic frequency is

$$
Q_{T E D}=K_{T E} \frac{\left(1+\omega_{0}^{2} / \omega_{T E}^{2}\right)}{\omega_{0} / \omega_{T E}},
$$

and the scaling constant is

$$
\omega_{T E}=\kappa /\left(C_{p} \rho(w / \pi)^{2}\right),
$$

$$
K_{T E}=C_{p} \rho / E T \alpha^{2} .
$$

Given the properties of $\mathrm{SiC}$ (Table 1 ) and the beam widths here $(5 \mu \mathrm{m}), \omega_{\mathrm{TE}} / 2 \pi=6 \mathrm{MHz}$. The relative distance between $\omega_{\mathrm{TE}}$ and the resonance $\omega_{0}$ yields large TED; minimum $Q_{T E D}$ would occur at $\omega_{\mathrm{TE}}=\omega_{0}$. Moving $\omega_{\mathrm{TE}}$ up (and $Q_{T E D}$ up) requires thinner beams, a capability yet to be developed in $\mathrm{SiC}$ fabrication technology. Conversely, $\omega_{\mathrm{TE}}$ for $\mathrm{Si}$, where thinner beams of $2 \mu \mathrm{m}$ are used, is $22 \mathrm{MHz}$, farther from the point of maximum TED. Improved $\mathrm{SiC}$ fab tolerances will permit higher Q. Frequency splits of $70.8 \mathrm{~Hz}$ (out of $88 \mathrm{kHz}$ ) and $161 \mathrm{~Hz}$ (out of $65.9 \mathrm{kHz}$ ) were measured on $\mathrm{SiC}$ and Si resonators, respectively. Electrostatic tuning of $k_{x}, k_{y}$, and $k_{x y}$ was also demonstrated (Figure 5), and tuned the frequency split below $12 \mathrm{~Hz}$. Damping mismatches $\Delta\left(\omega_{0} / 2 Q\right)$ were measured at $0.07 \mathrm{~Hz}$ for both devices.

Both $\mathrm{Si}$ and $\mathrm{SiC}$ gyros were also operated in open-loop rate mode. On a rate table, one of the $n=2$ vibratory modes was located and a function generator applied an input at half the resonant frequency. The amplitude of the drive and sense axis pickoffs were measured and demodulated, enabling separation of the quadrature signal. Rates up to $+/-200 \mathrm{deg} / \mathrm{sec}$ were applied, and the gyro response (demodulated sense amplitude divided by drive amplitude) is plotted in Figure $6(\mathrm{SiC})$, and Figure $7(\mathrm{Si})$. Scale factor depends on the frequency split (as described in the model equation in Figure 7) and the Bryan angular gain factor $A G$, which is typically between 0.6 and 0.8 for $n=2$ devices. The difference in slope between model and measurement in Figure 7 is therefore partially from $A G$, yielding an estimate of $A G=0.75$ for the $\mathrm{Si}$ gyros. The model also differs from the measurement because of demodulation phase error, and offset between function generator and resonance frequencies, errors that would largely be remedied by closed-loop electronics. These discrepancies did not permit a reliable estimate of $A G$ for $\mathrm{SiC}$ gyros. Scale factor varied inversely with frequency split as the electrostatic tuning was refined, agreeing with the ratemode model. Whole angle mode was not yet demonstrated, but is planned once electronics are completed.

\section{REFERENCES}

[1] D. D. Lynch, "Vibratory Gyro Analysis by Method of Averaging," in Second St. Petersburg Conference on Gyroscope Technology and Navigation, St. Petersburg, Russia (1995)

[2] R. Candler, et al. "Single wafer encapsulation of MEMS devices," IEEE Transactions on Advanced Packaging (2003)

[3] L. E. Luna, et al. "SiC MEMS technology based on wafer bonding and deep etching," in International Conference on Silicon Carbide and Related Materials, Washington, DC (2017)

[4] F. J. Kub, et al. "Whole Angle MEMS Gyroscope on Hexagonal Crystal Substrate." US Patent Application A1/20160341552, 24 November 2016.

\section{CONTACT}

*E. Cook, tel: +1-617-258-4123; ecook@draper.com

Table 1: Silicon carbide and silicon material properties

\begin{tabular}{|l|c|c|c|c|c|}
\hline Material & $C_{p}$ & $\rho$ & $E$ & $\kappa$ & $\alpha$ \\
\hline & $\mathrm{J} /(\mathrm{kg} \mathrm{K})$ & $\mathrm{kg} / \mathrm{m}^{3}$ & $\mathrm{GPa}$ & $\mathrm{W} /(\mathrm{m} \mathrm{K})$ & $\mathrm{ppm} / \mathrm{K}$ \\
\hline $\mathbf{S i C}$ & 750 & 3210 & 444 & 370 & 4.5 \\
\hline $\mathbf{S i}$ & 670 & 2330 & 165 & 140 & 2.5 \\
\hline
\end{tabular}

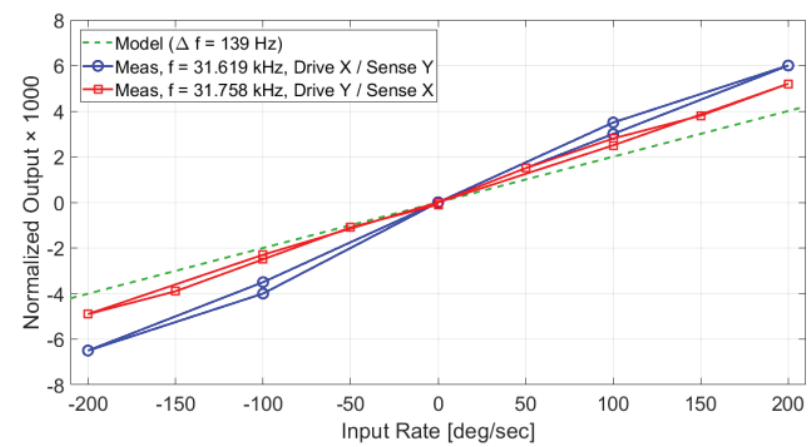

Figure 6: Rate mode gyroscope operation of the SiC device, driven on the $x$ mode (blue) and the y mode (red), with bias removed. Normalized output is the demodulated sense amplitude divided by drive amplitude. Scale factor was low because of the large frequency split.

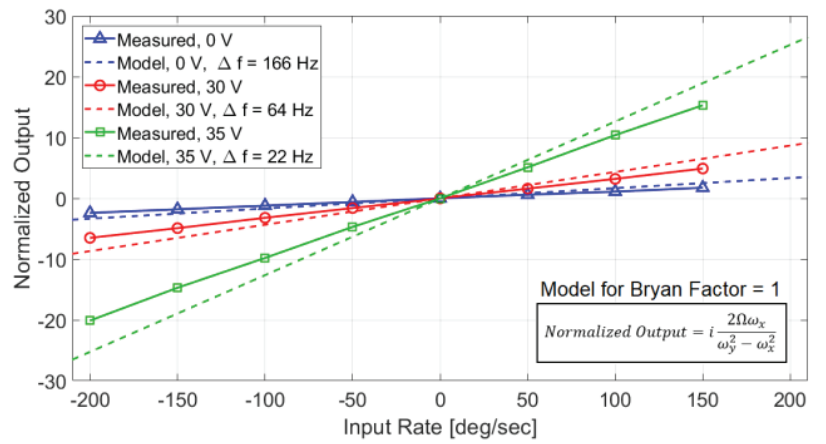

Figure 7: Rate mode gyroscope operation of the Si device, with bias removed. Gyro scale factor is increased (blue to green curve) by tuning the frequency split by applying the listed electrostatic tuning voltages to the sense axis. 